\title{
Vortex ring dynamics at a free surface
}

\author{
L. P. Bernal and J. T. Kwon \\ Department of Aerospace Engineering, University of Michigan, Ann Arbor, Michigan 48109-2140
}

(Received 8 November 1988; accepted 14 December 1988)

\begin{abstract}
The results of a flow visualization study of the evolution of vortex rings near a free surface are presented. The vortex rings were formed underwater with their axis parallel to the free surface one ring diameter below the surface. It is shown that vortex lines in the ring open during the interaction with the surface. The resulting flow field consists of vortex lines, beginning and terminating at the free surface. Data are reported on several features of the vortex ring evolution, which show a large reduction of the propagation speed and an oscillation of the vortex eccentricity as it propagates downstream after the interaction.
\end{abstract}

The dynamics of an underwater vortex ring moving parallel to the free surface is one of the simplest flow configurations in which the processes of vorticity cancellation and generation at a free surface can be studied. These flow processes play an important role in the dynamics of turbulent shear flows near a free surface. Recent studies of the interaction of a turbulent underwater jet with the free surface have shown the onset of surface swirls during the interaction. ${ }^{1.2}$ These swirls are associated with a vorticity vector at the free surface perpendicular to the surface. The present study was undertaken to investigate further the flow processes resulting in the introduction of surface vorticity.

The flow geometry is shown schematically in Fig. 1. The experiments were conducted in a free-surface water tank described in Ref. 1 . The vortex rings were generated at the exit of a reservoir tank by a short duration pressure pulse applied to the reservoir. The exit orifice on the reservoir tank had a circular cross section, $25.4 \mathrm{~mm}$ in diameter. A rounded contraction was located upstream of the exit orifice. The experiments were conducted with the exit orifice centerline located at approximately one ring diameter below the free surface. The vortex ring circulation was determined at each flow condition from velocity traces measured with a hot film probe at the exit plane of the reservoir. The circulation of the vortex ring was calculated using the expression

$$
\Gamma=\int_{0}^{T} \frac{U(t)^{2}}{2} d t
$$

where $T$ is the duration of the pressure pulse and $U(t)$ is the velocity at the centerline of the exit orifice. ${ }^{3}$ The underwater flow was visualized by adding fluorescent dye to the reservoir fluid and illuminating the flow with a thin sheet of laser light. In all flow pictures the illuminated plane is the plane of symmetry perpendicular to the free surface. The free-surface deformation was visualized by illumination with a collimated beam from a mercury arc lamp. The refracted beam at the free surface was viewed on a screen positioned at an angle of $45^{\circ}$, with respect to the water surface. Both the vortex ring cross section and the surface deformation were simultaneously recorded on the same photographic film or video image. Standard $35 \mathrm{~mm}$ photographic and video equipment were used to record the images. The video recordings of the vortex evolution were used to measure the time evolution of the various parameters described below.
A typical evolution of the vortex ring at a Reynolds number $\Gamma / v=7.4 \times 10^{3}$ and Froude number $\Gamma /\left(g a^{3}\right)^{1 / 2}=0.41$ is shown in Fig. 2. The photographs in this figure are simultaneous visualizations of the underwater flow and the surface deformation, each obtained on a different realization of the flow. Figure 2 (a) shows the initial evolution of the flow after formation of the vortex ring. A small depression of the free surface is observed above the vortex ring. Also, the free surface influences the motion of the vortex ring, causing a small deflection of the ring axis toward the free surface. The most striking feature of the interaction is evident in Figs. 2(b) and 2(c), which show the opening of vortex lines as the upper part of the ring interacts with the free surface. The upper half of the vortex ring with clockwise vorticity (CR) disappears from the cross-sectional view, while two distinct dark spots appear on the free surface. These are associated with vortex lines terminating at the free surface. ${ }^{4,5}$ The opening of vortex lines at the free surface can be viewed as a vortex tube reconnection process of the vorticity below the surface with its image above it. ${ }^{6-10}$ The interaction of the lower part of the vortex ring, that containing counterclockwise vorticity (CCR), with the free surface does not result in vortex tube reconnection. Rather, Fig. 2 (d) shows clockwise vorticity generated at the free surface rolling up in a secondary vortex. In the subsequent evolution the CCR core bounced back away from the free surface.

Several features of the interaction of the vortex ring with the free surface were measured in the video recordings of the evolution. The measurements of the extent of the interaction

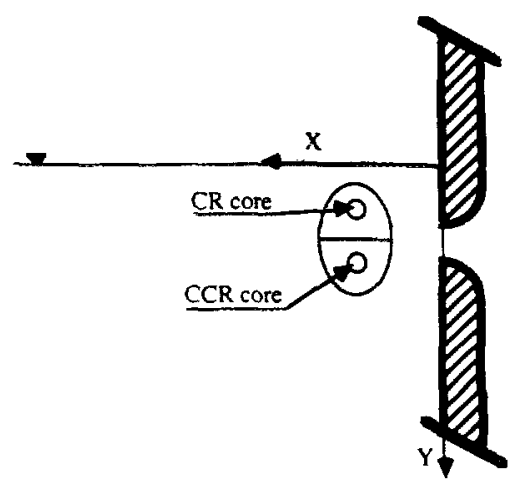

FIG. 1. A schematic diagram of flow geometry. $\mathbf{x}$, free-surface location. 

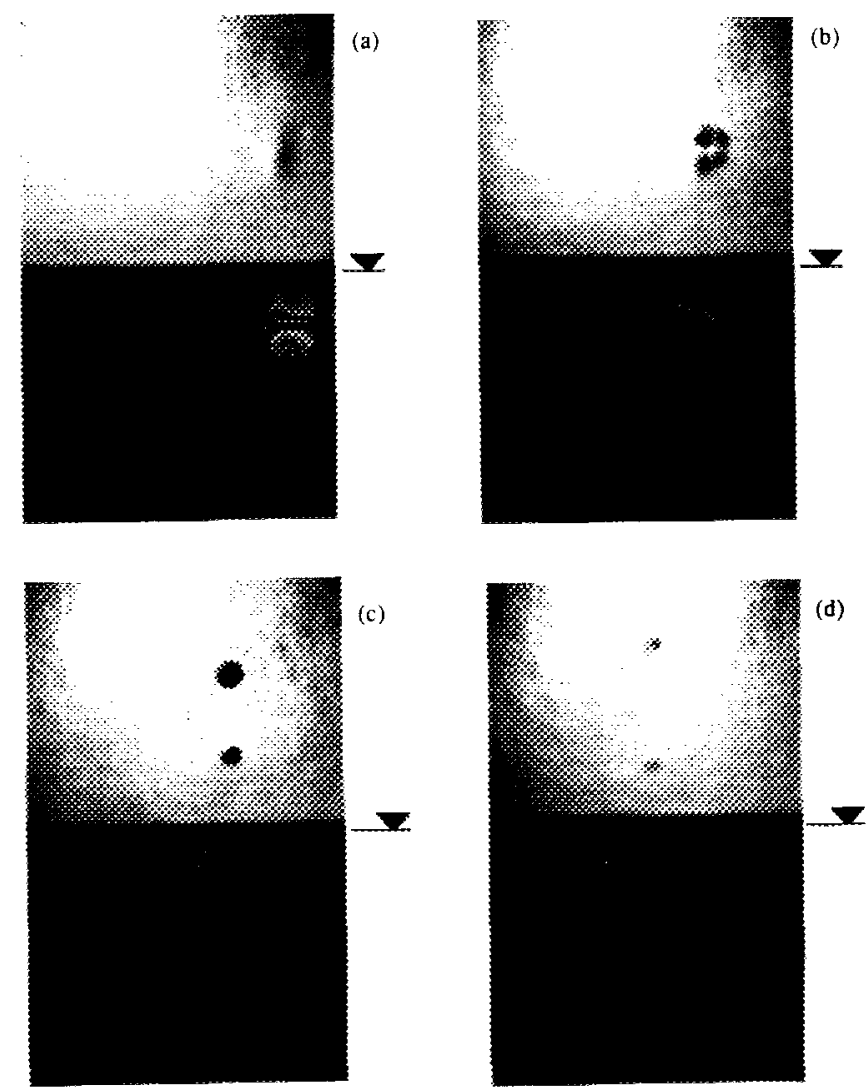

FIG. 2. A sequence of photographs showing the vortex ring evolution near the free surface. Ring motion is from right to left (see the text for further explanation). $\mathbf{I}$. free-surfacelocation; (a) $\Gamma t / a^{2} \approx 5$, (b) $\Gamma t / a^{2} \approx 13$, (c) $\Gamma t / a^{2} \approx 20$, and (d) $\Gamma t / a^{2} \approx 25$.

region as a function of vortex ring circulation are shown in Fig. 3. The extent of the interaction was characterized by measurements of the downstream location of the CR core when the vortex ring outline first reached the free surface, $X_{\text {, }}$, and by the downstream location of the CCR core at the point of closest proximity to the free surface, $X_{a}$. These two conditions define the approximate beginning and end of the interaction process, respectively. These locations and the extent of the interaction region normalized by the initial vortex ring diameter $a$ are plotted in Fig. 3 as a function of Reynolds number. It is apparent that the interaction occurs closer to the exit plane of the vortex ring as the Reynolds number is

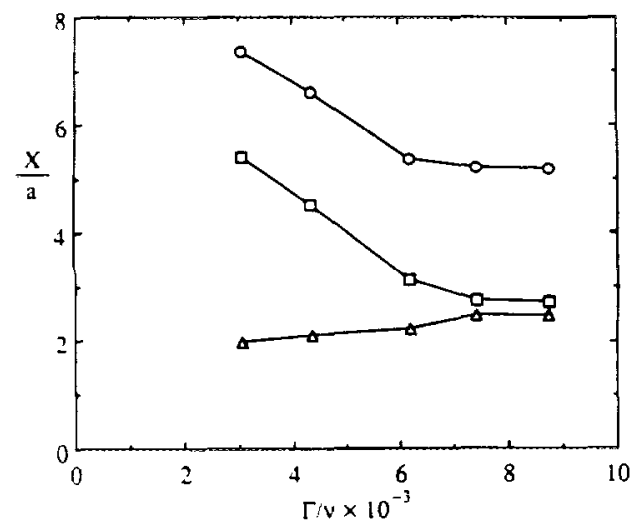

FIG. 3. The effect of Reynolds number on the extent of interaction. - $\square-$ $X, / a ;-\mathrm{O}_{-}, X_{v} / a ;-\Delta-,\left(X_{t}-X_{1}\right) / a$. increased. Also, the extent of the interaction region, $X_{c}-X_{s}$, increases slightly with Reynolds number. These results are in general agreement with the observations of Oshima and Asaka ${ }^{B}$ of the interaction of two vortex rings moving parallel to each other in air.

Detailed data on the evolution of vortex rings were obtained at a Reynolds number of $7.4 \times 10^{3}$ and Froude number $\Gamma /\left(g a^{3}\right)^{1 / 2}=0,41$. Figure 4 is a plot of the downstream location of the vortex ring cores normalized by the initial vortex ring diameter as a function of nondimensional time $\left(\Gamma t / a^{2}\right)$. At these flow conditions, and according to the criteria introduced above, the interaction starts at a nondimensional time of 10.3. The CR core disappears from the cross section at a nondimensional time of 12.6 , somewhat after the beginning of the interaction. There is a small difference in the $X$ location of the CR and CCR cores because of the deflection of the vortex ring toward the free surface. The path of the CCR core can be approximated by two straight lines of different slope, as shown in the figure. The slope change occurs at a nondimensional time of 20 . A least-squares fit to the data gives a nondimensional velocity $U a / \Gamma=0.36$ before the interaction and $U a / \Gamma=0.066$ after the interaction. The large reduction in propagation speed can be attributed to the combined effect of the change in radius of curvature of the vortex lines as well as the clockwise vorticity generated at the free surface.

Figure 5 is a plot of the normalized distance of the vortex cores to the free surface, $Y / a$, as a function of nondimensional time. Also plotted in Fig. 5 is the normalized distance between vortex cores measured on the free surface, $D / a$. Before the reconnection the vortex cores approach the free surface at a speed typically one order of magnitude lower than the propagation speed of the ring. The cores also approach each other, suggesting an elongated vortex core. The vortex cores are first observed on the free surface at a nondimensional time of 13.0, after the vortex tube reconnection process is completed. The distance between vortex cores on the free surface and the distance of the CCR core to the free surface oscillate after the interaction. The two oscillatory motions are out of phase, suggesting an oscillation of the opened vortex ring eccentricity. The oscillatory motion is readily observed at times longer than those shown in Fig. 5. The period of the oscillation, $t_{\rho}$, is estimated as $\Gamma t_{\rho} / a^{2} \approx 50$. This value compares well with the theoretical calculation for

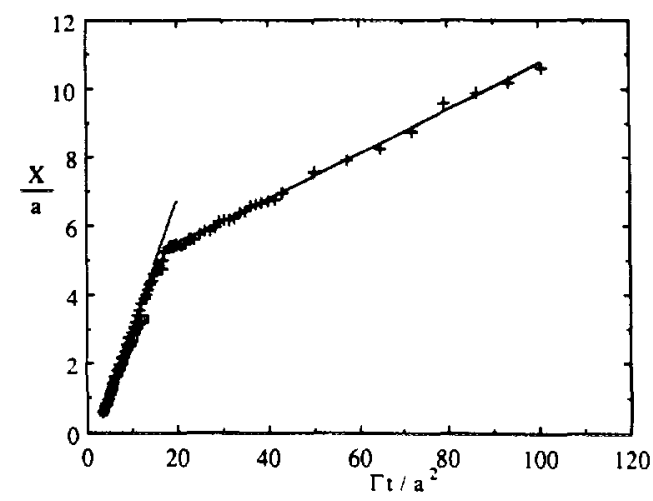

FIG. 4. The downstream location of vortex ring cores as a function of time. $\square, X_{\mathrm{CR}} / a ;+, X_{\mathrm{CCH}} / a ;--$, least-squares fit to CCR core location. 


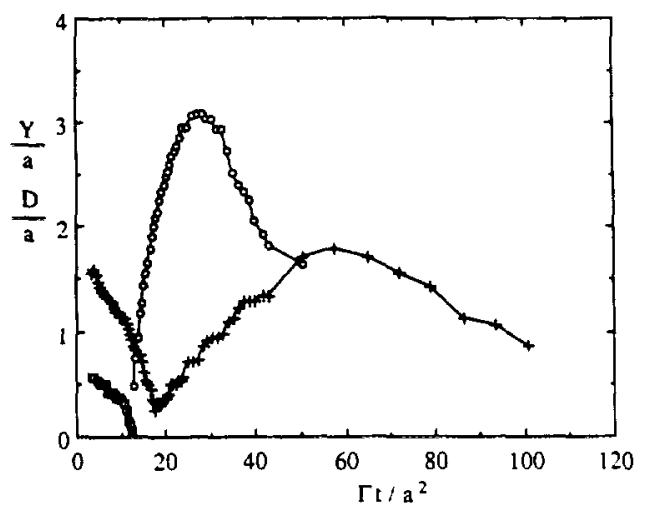

FIG. 5. The distance of vortex cores to the free surface as a function of time. ๑, $Y_{\mathrm{CK}} / a ;+, Y_{\mathrm{CCR}} / a ;-\mathrm{O}, D / a$.

small amplitude oscillations (mode $n=2$ ) of a thin-cored vortex ring having the measured diameter and propagation speed after the interaction and the initial circulation," $\Gamma t_{p} / a^{2}=41$.

An important parameter influencing the dynamics of vortex rings is the core diameter $\delta$. An approximate measurement of this parameter can be obtained from the diameter of the dark spots on the free surface after the reconnection process is completed. ${ }^{4.5}$ The estimated value at these conditions is $\delta / a=0.56$. This value gives an estimate for the propagation speed of the vortex ring, ${ }^{12} U a / \Gamma=0.38$, in good agreement with the measured initial speed of the vortex ring. The core diameter also determines the duration of the vortex tube reconnection process. ${ }^{9.13}$ For these measurements the duration of the vortex reconnection process is estimated as the time difference $t$, from the start of the interaction to the time the vortex cores are first observed on the free surface, which gives $\Gamma t_{r} / a^{2}=2.7$. This result is consistent with the scaling proposed by Schatzle ${ }^{9}$ for the interaction of two colliding vortex rings:

$$
\Gamma t_{r} / a^{2} \approx \frac{1}{2}(\delta / a)^{2} \ln (\Gamma / v)=1.4 .
$$

In summary, the interaction of the free surface with a vortex ring moving parallel to the surface is characterized by two very different interaction processes. The first interaction results in vortex tube reconnection similar to the reconnection process of colliding vortex rings. In contrast, the second interaction results in the generation of vorticity at the free surface in a manner reminiscent of a solid surface, ${ }^{14}$ or at a density interface. ${ }^{15}$ The role of unsteady vorticity generation at the free surface and surface contaminants on these processes is currently under investigation.

\section{ACKNOWLEDGMENTS}

We would like to thank Professor W. W. Willmarth, Professor G. Tryggvason, and Professor W. J. A. Dahm for many stimulating discussions and $\mathbf{K}$. Madnia for assistance with the flow facility.

This research was sponsored by the Office of Naval Research, Contract No. N000184-86-K-0684 under the URI Program for Ship Hydrodynamics.

'L. P. Bernal and K. Madnia, in Proceedings of the 17th Symposium of Naval Hydrodynamics, in press.

'L. P. Bernal and J. T. Kwon, submitted to Exp. Fluids.

${ }^{3}$ N. Didden, J. Appl. Math. Phys. 30, 101 (1979).

${ }^{4} M$. V. Berry and J. V. Hajnal, Opt. Acta 30, 23 (1983),

'M. H. Sterling, M. Gorman, P. J. Widmann, S. C. Coffman, J. Strozier, and R. M. Kiehn, Phys. Fluids 30, 3624 (1987).

${ }^{6} T$. Kambe and T. Takao, J. Phys. Soc. Jpn. 31, 591 (1971).

${ }^{7}$ T. Fohl and J. S. Turner, Phys. Fluids 18, 433 (1975).

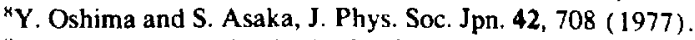

${ }^{4} P$. R. Schatzle, Ph.D. thesis, California Institute of Technology, 1987.

"Y. Oshima and N. Izutsu, Phys. Fluids 31, 2401 (1988).

"S. E. Widnall and J. P. Sullivan, Proc. R. Soc. London Ser. A 332. 335 (1973).

''H. Lamb, Hydrodynamics (Cambridge U.P., Cambridge, 1932), Sec. 163

${ }^{1.3}$ W. T. Ashurst and D. I. Meiron, Phys. Rev. Lett. 58, 1632 (1987).

14J. D. A. Walker, C. R. Smith, A. W. Cerra, and T. L. Doligalski, J. Fluid Mech. 181, 99 (1987).

I'W. J. A. Dahm, C. M. Scheil, and G. Tryggvason, to appear in J. Fluid Mech.

\title{
Log-corrected energy spectrum and dimension of attractor in two-dimensional turbulence
}

\author{
Koji Ohkitani \\ Department of Physics, Faculty of Science, Kyoto University, Kyoto 606, Japan
}

(Received 8 November 1988; accepted 5 December 1988).

The dimension of the attractor in two-dimensional turbulence is estimates qualitatively by using Kraichnan's log-corrected energy spectrum. It is found to be identical to the leading order with rigorous upper bounds, obtained by Constantin, Foias, and Temam [Physica D 30 , 284 (1988) ], which also include log factors.

The energy spectrum in the (enstrophy) inertial subrange of two-dimensional turbulence has long been controversial. We will denote by $E(k)$ the energy spectrum at wavenumber $k$. The first, and probably the simplest, form has been given by the dimensional argument in the enstrophy cascade theory as'

$$
E(k) \sim \eta^{2 / 3} k^{-3}, \text { for } k_{0} \ll k \ll k_{d},
$$

